\title{
EM Wave Scattering by Many Small Impedance Particles and Applica- tions to Materials Science
}

\author{
Alexander G. Ramm*
}

Department of Mathematics, Kansas State University, Manhattan, KS 66506, USA

\begin{abstract}
An explicit formula is derived for the electromagnetic (EM) field scattered by one small impedance particle $D$ of an arbitrary shape. If $a$ is the characteristic size of the particle, $\lambda$ is the wavelength, $a<<\lambda$, and $\zeta$ is the boundary impedance of $D,[N,[E, N]]=\zeta[N, H]$ on $S$, where $S$ is the surface of the particle, $N$ is the unit outer normal to $S$, and $E$, $H$ is the EM field, then the scattered field is $E_{S C}=\left[\nabla g\left(x, x_{1}\right), Q\right]$. Here $g(x, y)=\frac{e^{i k|x-y|}}{4 \pi|x-y|}, k$ is the wave number, $x_{1} \in D$ is an arbitrary point, and $Q=-\frac{\zeta|S|}{i \omega \mu} \tau \nabla \times E_{0}$, where $E_{0}$ is the incident field, $|S|$ is the area of $S, \omega$ is the frequency, $\mu$ is the magnetic permeability of the space exterior to $D$, and $\tau$ is a tensor which is calculated explicitly. The scattered field is $O\left(|\zeta| a^{2}\right)>>O\left(a^{3}\right)$ as $a \rightarrow 0$ when $\lambda$ is fixed and $\zeta$ does not depend on $a$. Thus, $\left|E_{s c}\right|$ is much larger than the classical value $O\left(a^{3}\right)$ for the field scattered by a small particle. It is proved that the effective field in the medium, in which many small particles are embedded, has a limit as $a \rightarrow 0$ and the number $M=M(a)$ of the particles tends to $\infty$ at a suitable rate. This limit solves a linear integral equation. The refraction coefficient of the limiting medium is calculated analytically. This yields a recipe for creating materials with a desired refraction coefficient.
\end{abstract}

Keywords: Electromagnetic wave scattering, small impedance body, scatterer of an arbitrary shape, EM wave scattering by many small bodies, creating materials with a desired refraction coefficient.

\section{INTRODUCTION}

Let $D \subset \mathbb{R}^{3}$ be a bounded domain with a connected smooth boundary $S, D^{\prime}:=\mathbb{R}^{3} \backslash D, k^{2}=$ const $>0$ is the wave number, $\omega>0$ is frequency, the boundary impedance $\zeta=$ const, $\operatorname{Re} \zeta \geq 0, \varepsilon>0$ and $\mu>0$ are dielectric and magnetic constants, $\varepsilon^{\prime}=\varepsilon+i \frac{\sigma}{\omega}, \sigma=$ const $\geq 0$ is the conductivity of $D^{\prime}, x \in D^{\prime}, r=|x|, N$ is the unit normal to $S$ pointing into $D^{\prime}$.

Let us assume that the electrical field $E=E_{0}+e$, where $E_{0}$ is the incident field and $e$ is the scattered field. Then $e$ solves the problem:

$$
\begin{aligned}
& \nabla \times e=i \omega \mu h, \nabla \times h=-i \omega \varepsilon^{\prime} h \text { in } D^{\prime} \\
& r\left(e_{r}-i k e\right)=o(1), r \rightarrow \infty \\
& {[N,[e, N]]-\frac{\zeta}{i \omega \mu}[N, \nabla \times e]=-f \text { on } S .}
\end{aligned}
$$

Here $f=\left[N,\left[E_{0}, N\right]\right]-\frac{\zeta}{i \omega \mu}\left[N, \nabla \times E_{0}\right]$ is a given smooth tangential field on $S, H=\frac{\nabla \times E}{i \omega \mu}, h=\frac{\nabla \times e}{i \omega \mu}, \varepsilon^{\prime}$ and $\mu$ are dielectric and magnetic constants of the medium $D^{\prime}$, $[A, B]=A \times B$ is the cross product of two vectors, $A \cdot B$ is their scalar product. Problem (1)-(3) is the scattering problem for electromagnetic (EM) waves by an impedance

*Address correspondence to this author at the Department of Mathematics, Kansas State University, Manhattan, KS 66506, USA;

E-mail: ramm@math.ksu.edu body $D$ of an arbitrary shape. This problem has been discussed in [1], where the uniqueness of its solution has been proved. Explicit formula for the plane EM wave scattered by a small impedance body $(k a=1, a$ is the characteristic size of this body) of an arbitrary shape is derived in [1]. There one can also find a solution to manybody scattering problem in the case of small impedance particles (bodies) of an arbitrary shape.

A few historical remarks are in order. The theory of wave scattering by small bodies was originated by Rayleigh in 1871. He understood that the main term in the scattered field is the dipole radiation. How to calculate this radiation, in other words, how to calculate the induced dipole moment on a small body of an arbitrary shape, Rayleigh did not show. This was done nearly 100 years later by the author, see [2]. In 1908 G.Mie published a method for solving scattering problems for well conducting spherical particles using separation of variables in the spherical coordinates. His method works also for spherical impedance particles, but does not work for particles of an arbitrary shape for which separation of variables cannot be used. Smallness of the particle is not required by the Mie's method.

There were no analytical methods for solving EM wave scattering problems for small bodies of an arbitrary shape. No explicit formulas for the scattered fields were obtained by other authors for small bodies of an arbitrary shape. Such methods for acoustic and EM waves were developed in the monograph [1].

Let us highlight the novel points in our theory: 
a) For one small impedance particle of an arbitrary shape an analytic formula for the scattered field is derived; this formula is asymptotically exact as $a \rightarrow 0$; the scattering amplitude is $O(|\zeta||S|)$. Assuming $|S|=O\left(a^{2}\right)$ and $\zeta=O\left(a^{-\kappa}\right), \kappa \in[0,1)$ is a constant, one obtains that the scattering amplitude is $O\left(a^{2-\kappa}\right)>>O\left(a^{3}\right)$, where $O\left(a^{3}\right)$ is the value of the scattering amplitude in the classical theory. This is a new physical phenomenon. If the particle is perfectly conducting, the corresponding scattering amplitude is $O\left(a^{3}\right)$ for small $a$.

b) In the case of many small impedance particles a method for solving the EM wave scattering problem is developed (see formulas (10)-(12)) and the limiting integral equation (see formula (13)) is derived for the limiting effective field in the medium where very many small impedance particles are embedded.

c) These results are used to formulate a method for creating materials with a desired refraction coefficient, see Section 5.

These results do not intersect with the results published by other authors.

\section{FORMULA FOR THE SOLUTION OF THE EM WAVE SCATTERING BY ONE SMALL BODY}

Our first result is the following explicit formula for the scattered field:

$e=\left[\nabla g\left(x, x_{1}\right), Q\right], g(x, y):=\frac{e^{i k|x-y|}}{4 \pi|x-y|}$

which is asymptotically, as $a \rightarrow 0$, exact. The quantity $Q$ in formula (4) is given by the following formula:

$Q=-\frac{\zeta|S|}{i \omega \mu} \tau \nabla \times E_{0}$

where $E_{0}$ is the incident field, the tensor $\tau$ is defined as follows:

$\tau=I-b, b=\left(b_{j m}\right)=\frac{1}{|S|} \int_{S} N_{j}(s) N_{m}(s) d s$

and $d s$ is an element of the surface area. In formula (5) the terms of the higher order of smallness, as $a \rightarrow 0$, are neglected.

When we write $a \rightarrow 0$, it means that $\lambda$ is fixed: the physical meaning has the ratio $a / \lambda$.

Formulas (4)-(6) are proved in [1]. In their derivation a new representation of the scattered field $e$ is used:

$e=\nabla \times \int_{S} g(x, t) J(t) d t, \int_{S} J(t) d t:=Q$

where $J$ is a tangential to $S$ field. One can find $e$ of this form if $k^{2}$ is not a Dirichlet eigenvalue of the Laplacian in $D$. If $k^{2}$ is an eigenvalue of the Laplacian in $D$, then one can use $g_{\rho}(x, y)$ in place of $g(x, y)$. Here $g_{\rho}(x, y)$ is the Green's function of the Helmholtz operator in the exterior of $B(0, \rho)$, the ball centered at the origin and of radius $\rho$. The $g_{\rho}(x, y)$ satisfies the Dirichlet condition on the boundary of $B(0, \rho)$ and the radiation condition at infinity. The small number $\rho>0$ is chosen so that $k^{2}$ is not a Dirichlet eigenvalue of the Laplacian in $D \backslash B(0, \rho)$. Such a ball can always be found and $\rho>0$ can be chosen as small as one wishes.

Let us discuss formulas (4)-(6). The choice of $x_{1} \in D$ in formula (4) is not important since $a$ is small. If $D$ is centrally symmetric body, then one can take as $x_{1}$ its symmetry center. Formula (5) shows that the scattering amplitude $A$, $A=O(|\zeta||S|)=O\left(a^{2}\right)$ if $\zeta$ does not depend on $a$. The classical dependence of the scattering amplitude on $a$ is $O\left(a^{3}\right)$, which is much smaller than $O\left(a^{2}\right)$ as $a \rightarrow 0$.

This fact might be of physical interest in applications.

Formula (6) gives explicitly the dependence of the scattered field on the shape of the small body.

The main physical (and mathematical) idea, used in [1] for the derivation of the above results is the reduction of the scattering problem for a small particle to finding just one quantity $Q$ rather than a boundary function. In the next Section the many-body scattering problem is discussed.

\section{EM WAVE SCATTERING BY MANY SMALL IMPEDANCE PARTICLES}

Let us formulate our basic results for EM wave scattering by many small impedance particles $D_{m}$, $1 \leq m \leq M$, distributed in a bounded domain $\Omega$. Let $x_{m} \in D_{m}$ be some points. Assume that the number $\mathcal{N}(\Delta)$ of small bodies (or points $x_{m}$ ) in any sub-domain $\Delta \subset \Omega$ is given by the formula

$\mathcal{N}(\Delta)=\frac{1}{a^{2-\kappa}} \int_{\Delta} N(x) d x(1+o(1)), \quad a \rightarrow 0$

where $N(x) \geq 0$ is a continuous in $\Omega$ function, $\kappa \in[0,1)$ is a parameter, and the boundary impedance of the $m$-th small particle is $\zeta_{m}:=h\left(x_{m}\right) / a^{\kappa}$, where $h(x)$ is a continuous function in $\Omega$, such that Reh $\geq 0$, and $x_{m} \in D_{m}$ is an arbitrary point.

The choice $\zeta=h(x) a^{-\kappa}$ is not dictated by physical laws. As we mentioned earlier, the choice of $\zeta$ is made by the experimentalist as he wishes.

The only physical restriction on the boundary impedance is the relation $\operatorname{Re} \zeta \geq 0$, that is, $\operatorname{Reh}(x) \geq 0$.

The restriction for the parameter $\kappa \in[0,1)$ is of technical nature and is not related to a physical law.

The functions $N(x), h(x)$ and the parameter $\kappa$ can be chosen by the experimentalist as he/she wants.

Our main physical assumption is:

$a<<d<<\lambda$

where $d$ is the minimal distance between neighboring small particles.

Our second result is the following formula for the solution of many-body EM wave scattering problem: 
$E(x)=E_{0}(x)+\sum_{m=1}^{M}\left[\nabla g\left(x, x_{m}\right), Q_{m}\right], \quad a \rightarrow 0$

where $Q_{m}$ are defined by the formula:

$Q_{m}=-\frac{\zeta_{m}|S|}{i \omega \mu} \tau \nabla \times E_{e m}$

Here we assumed for simplicity that the particles have the same shape, so tensor $\tau$ does not depend on $m$, and $E_{e m}$ is the effective field acting on the $m-$ th particle. This field is defined by the formula:

$E_{\text {em }}=E_{0}\left(x_{m}\right)+\sum_{j=1, j \neq m}^{M}\left[\nabla g\left(x_{m}, x_{j}\right), Q_{j}\right]$

Formulas (10)-(11) lead to the linear algebraic system for finding the unknown quantities $E_{m}:=E_{e m}$ :

$E_{m}=E_{0}\left(x_{m}\right)-\frac{c_{S}}{i \omega \mu} \sum_{j=1, j \neq m}^{M}\left[\nabla g\left(x_{m}, x_{j}\right), \tau \nabla \times E_{j}\right] h_{j} a^{2-\kappa}, \quad 1 \leq$ $m \leq M$

where $c_{S}>0$ is the constant in the formula $|S|=c_{S} a^{2}$.

Our third result is the following integral equation for the limiting effective field in $\Omega$ :

$E(x)=E_{0}(x)-\frac{c_{S}}{i \omega \mu} \nabla \times \int_{\Omega} g(x, y) \tau \nabla \times E(y) h(y) N(y) d y$

where $N(y)$ is defined in formula (7) and the limit is taken as $a \rightarrow 0$. The existence of this limit is proved in [1].

Equation (13) is equivalent to the following local differential equation:

$\nabla \times \nabla \times E(x)=k^{2} E(x)-\frac{c_{S}}{i \omega \mu} \nabla \times(h(x) N(x) \tau \nabla \times E(x))$

as one can check by applying the operator $\nabla \times \nabla \times$ to equation (13), see the details in [1].

Let us assume that $\tau$ is proportional to a diagonal matrix I. This happens, for example, if the particles are balls of radius $a$, in which case $\tau=\frac{2}{3} I$, as one can easily verify.

Then equation (14) takes the form:

$\nabla \times \nabla \times E(x)=\frac{k^{2} E(x)}{1+\frac{2 c_{S}}{3 i \omega \mu} h(x) N(x)}-\frac{2 c_{S}}{3 i \omega \mu} \cdot \frac{[\nabla(h(x) N(x)), \nabla \times E(x)]}{1+\frac{2 c_{S} S}{3 i \omega \mu} h(x) N(x)}$

\section{PHYSICAL INTERPRETATION OF FORMULA (15)}

To interpret physically formula (15), consider the Maxwell's equations:

$\nabla \times E(x)=i \omega \mu H, \nabla \times H(x)=-i \omega \varepsilon^{\prime} H$

where $\mu=\mu(x)$, apply the operator $\nabla \times$ to the first equation and then use the second one. This yields:

$\nabla \times \nabla \times E(x)=K^{2} E(x)+\left[\frac{\nabla \mu(x)}{\mu(x)}, \nabla \times E(x)\right]$

where $K^{2}=\omega^{2} \mu(x) \varepsilon^{\prime}=k^{2} \frac{\mu(x) \varepsilon^{\prime}}{\mu_{0} \varepsilon_{0}}, k^{2}=\omega^{2} \varepsilon_{0} \mu_{0}$, and $\mu_{0}, \varepsilon_{0}$ are the parameters of the free space. Let $n(x):=\frac{\mu(x) \varepsilon \prime}{\mu_{0} \varepsilon_{0}}$.

Comparing formulas (15) and (17) one concludes that the limiting medium, obtained by the embedding of many small impedance particles, has the new refraction coefficient: $n(x)=\frac{n_{0}(x)}{\left(1+\frac{2 c_{S}}{3 i \omega \mu} h(x) N(x)\right)^{1 / 2}}, n_{0}(x):=\left(\frac{\varepsilon^{\prime} \mu}{\varepsilon_{0} \mu_{0}}\right)^{1 / 2}$

and the new magnetic permeability:

$\mu(x)=\left(1+\frac{2 c_{S}}{3 i \omega \mu} h(x) N(x)\right)^{-1}$

On formula (18) our recipe for creating materials with a desired refraction coefficient is based. This recipe is discussed in the next Section.

\section{A RECIPE FOR CREATING MATERIALS WITH A DESIRED REFRACTION COEFFICIENT}

Let us rewrite formula (18) as

$n(x)=\frac{n_{0}(x)}{\left(1-i c_{1} h(x) N(x)\right)^{1 / 2}}$

where $\quad c_{1}:=\frac{2 c_{S}}{3 \omega \mu}>0$ and $h=h_{1}+i h_{2}, h_{1} \geq 0$. The functions $h(x)$ and $N(x) \geq 0$ are at the disposal of the experimentalist, as was mentioned earlier. By choosing these functions properly, one can get any desired refraction coefficient which has the property $\operatorname{Im} n(x) \geq 0$. One rewrites formula (20) as follows:

$n(x)=n_{0}(x)\left(1-i c_{1} h(x) N(x)\right)^{-\frac{1}{2}}=n_{0}(x)(1+$ $\left.c_{1} h_{2}(x) N(x)-i c_{1} h_{1}(x) N(x)\right)^{-\frac{1}{2}}$

Let $z$ be a complex number. Define $z^{1 / 2}=|z|^{1 / 2} e^{i \phi / 2}$, where $\phi$ is the argument of $z, 0 \leq \phi<2 \pi$. By choosing $h_{2}<0$ so that $1+c_{1} h_{2}(x) N(x)$ is small and choosing $h_{1} \geq 0$ suitably, one can make $|n(x)|$ to be any desired nonnegative function. The argument $\phi$ of the expression

$$
1+c_{1} h_{2}(x) N(x)-i c_{1} h_{1}(x) N(x)
$$

can be made arbitrary by choosing $h_{1} \geq 0$ and $h_{2} \in$ $(-\infty, \infty)$ suitably.

For example, assume that $n_{0}>0, h_{1}>0$ is small and $1+c_{1} h_{2}(x) N(x)>0$. Then the $\phi=2 \pi-2 \delta$, where $\delta$ is arbitrarily small, $\phi / 2=\pi-\delta$ and $n(x)=|n(x)| e^{-i(\pi-\delta)}$. Thus, $\operatorname{Re} n(x)<0, \quad \operatorname{Im} n(x) \geq 0, \quad$ and $\operatorname{Im}$ $n(x)=|n(x)| \sin \delta$ can be made as small as one wishes if $\delta$ is sufficiently small. Therefore, one gets a material with negative refraction and negligible losses.

Similarly, using formula (19), one can change magnetic permeability in a desired direction by embedding in a given medium many small impedance particles with the suitable boundary impedances.

\section{CONFLICT OF INTEREST}

The author confirms that this article content has no conflicts of interest.

\section{ACKNOWLEDGEMENTS}

Declared none. 


\section{REFERENCES}

[1] Ramm AG. Scattering of acoustic and electromagnetic waves by small bodies of arbitrary shapes: Applications to creating new engineered materials, New York: Momentum Press 2013.
[2] Ramm AG. Wave scattering by small bodies of arbitrary shapes. Singapore: World Sci Publishers 2005.

Received: December 03, 2014

Revised: January 24, 2015

Accepted: February 25, 2015

(C) Alexander G. Ramm; Licensee Bentham Open.

This is an open access article licensed under the terms of the Creative Commons Attribution Non-Commercial License (http://creativecommons.org/licenses/by-nc/3.0/) which permits unrestricted, non-commercial use, distribution and reproduction in any medium, provided the work is properly cited. 